\title{
The ketamine crisis: Does South Africa have a plan B?
}

\author{
S Wall, ${ }^{1}$ MB ChB, DipPEC (SA), FCS (SA), MMed (Surgery); V Bangalee, ${ }^{2}$ BPharm, MPharm, PhD \\ ${ }^{1}$ Pietermaritzburg Burn Service, Pietermaritzburg Metropolitan Department of Surgery, South Africa; and School of Clinical Medicine, \\ Nelson R Mandela School of Medicine, University of KwaZulu-Natal, Pietermaritzburg, South Africa \\ ${ }^{2}$ Discipline of Pharmaceutical Sciences, School of Health Sciences, University of KwaZulu-Natal, Durban, South Africa
}

Corresponding author: S Wall (shelley_wall@hotmail.com)

South Africa (SA) has a high incidence of deaths from trauma and injuries. Trauma has been identified as one part of the quadruple burden of disease afflicting the country. This article is concerned with the management of burns, which $3 \%$ of the population suffer from annually. Ketamine, acknowledged for its versatility and safety profile, remains a critical component in the medical arsenal of anaesthesiologists and clinicians treating both acute and chronic pain. In the management of burn-injured patients in particular, ketamine is the cornerstone of many analgesia protocols. However, issues pertaining to shortages of this medicine in SA warrant concern and discussion, particularly in view of the high reliance of doctors on ketamine for first-line procedural analgesia in the management of burns in both adult and paediatric patients. This article attempts to highlight the issues related to ketamine shortages, which often have significant clinical, safety, operational and research implications.

S Afr Med J 2019;109(12):911-913. https://doi.org/10.7196/SAMJ.2019.v109i12.14188

In excess of 1 million people suffer from burns in Africa annually. ${ }^{[1]}$ Across the African continent, burns account for $18 \%$ of hospital admissions and have a mortality rate of $6-10 \% \cdot{ }^{[1]}$ According to a 2017 Medical Research Council report, ${ }^{[2]}$ South Africa (SA)'s annual burn mortality rate of $8.5 / 100000$ is substantially above the world average of 5/100 000. The same report stated that $3 \%$ of the SA population suffer from burns annually, and that of these burns, $20 \%$ are classified as moderate to severe. Moreover, 161 children are severely burned in accidents in the home every month in SA, and because of inadequacies in the burn care system, 6 of these children will die. A World Health Organization report on burns in March $2018^{[3]}$ claimed that annually in SA, USD26 million is spent on burn care for patients burned in cooking incidents from kerosene (paraffin) stoves. ${ }^{[3]}$ The socioeconomic impact of this situation is worsened by indirect costs due to inability of these patients to return to work, resulting in their requiring disability grants, and their need for prolonged care for deformities caused by the burns.

\section{Defining the problem}

From the point of initial injury and throughout rehabilitation, the management of pain in burn patients is an area of major concern, with several clinicians attesting to burn pain being the most difficult of any of the acute pain aetiologies to treat. ${ }^{[4]}$ In addition to pain related to the burn, the curative and rehabilitative therapies compound the difficulty of pain control, as these interventions, which include dressing changes, excision and grafting, and physical therapy, cause pain that is often equivalent to or worse than the pain of the initial burn injury. ${ }^{[4]}$ Studies have shown that good pain management and control is associated with better wound healing, sleep, participation in activities of daily living, quality of life and recovery. ${ }^{[5,6]}$ For the above reasons, pain management forms the foundation of burn care.

The benefits of ketamine are numerous. Since its first clinical use in 1970, ketamine has continued to remain a critical component of the medical arsenal of anaesthesiologists and clinicians treating both acute and chronic pain, in particular finding a growing use in the management of adult and paediatric burns. ${ }^{[7]}$ Over the past two decades, several studies have investigated the additional antidepressant and anti-inflammatory effects of ketamine. ${ }^{[7-9]}$ The appeal of ketamine in the clinical setting is its versatility as a result of its unique ability to induce three clinical outcomes, i.e. narcosis, analgesia and amnesia. There is currently no other medicine used in clinical practice that can provide these three effects simultaneously. ${ }^{[7]}$ Apart from its rapid onset of action, it does not induce hypotension and there is minimal risk of airway loss because the pharyngeal and laryngeal reflexes are only slightly impaired. It also rarely induces bronchospasm, which makes it particularly useful in asthmatic patients as well as in children. Its increasing portfolio of use coupled with its safety profile have cemented its place in a plethora of management protocols in the South African Standard Treatment Guidelines. ${ }^{[10,11]}$

In the operating theatre, ketamine can be used for the induction and maintenance of anaesthesia. Because it does not induce hypotension, it is especially useful in cases where haemodynamic control is required. ${ }^{[12]}$ Ketamine is also used in various settings outside the operating room, such as procedural sedation for dressing changes, splinting, line changes and fracture reductions. Ketamine shortages therefore pose a threat to doctors and patients alike in the SA healthcare system, demanding national attention from all those practising in the field.

The impact of ketamine shortages is far reaching, as this drug is the safest, most practical option for procedural sedation for minor procedures in wards and outpatient departments in hospitals with either no theatre facilities or limited theatre time. When it is not possible to do simple procedures safely in the ward, they need to be done in theatre. Theatre time is a scarce and costly resource in our environment.

This dilemma ultimately affects the quality of patient care, as every situation in which ketamine is out of stock has a measurable human impact. From the perspective of patients and their families, 
medicine shortages result in wasted time and work days, money lost on pointless travel, poor retention and follow-up for chronic diseases, untreated diseases, and most importantly a loss of confidence in the public health system. ${ }^{[13]}$

At the bedside, doctors' frustration due to medicine shortages is shared by pharmacists, who are required to make complex decisions and provide recommendations to doctors based on a limited availability of options. The failing balance of supply (pharmacist) and demand (doctor) often results in strained relationships between these two members of the healthcare team in the clinical setting. Hospital pharmacists are required to find alternatives, leading to the administration of second- and third-line medicines that may be inferior in quality or outcome. In the case of ketamine specifically, alternative treatments may not be readily available and in addition may require additional training and resources for their safe use. Alternative regimens further raise the risk of dosage errors if healthcare practitioners are not familiar with the dosing and administration required for these products. This lack of training and knowledge associated with using alternatives leads to adverse effects, unforeseen complications, and effectiveness issues that negatively affect costs and place an added strain on an already overburdened healthcare budget. From a clinical and ethical standpoint, shortages of a medicine like ketamine, which has multiple indications in the healthcare sector, require tough decisions to be made on which patient populations will be given priority for a medicine that is in short supply.

\section{Alternatives to ketamine}

One potential alternative to ketamine is methoxyflurane. It is a short-acting inhalational analgesic agent with a rapid onset of action. The analgesic effects manifest after $6-8$ breaths, and once inhalation is ceased, the analgesic effects wear off within a few minutes. It has been well described in the management of acute pain and a variety of procedures including dressing changes and dental procedures. Although studies in the adult population predominate, there are studies proving it to be safe and efficacious in the paediatric population with minimal side-effects. ${ }^{[14]}$ One of the disadvantages of methoxyflurane is that it is currently not widely available in the public health sector. It is more expensive than ketamine, and the majority of doctors are reluctant to use medications that they are unfamiliar with, which also limits its use.

Other alternatives for procedural sedation, such as the combination of propofol and fentanyl, are also limited by lack of availability in certain state facilities. The safety of such combinations in the hands of healthcare workers inexperienced in procedural sedation, and in view of the lack of monitoring equipment in these facilities, is questionable.

\section{Why is there a ketamine shortage?}

The reasons for the ketamine shortage are not entirely clear. Some of the global reasons provided by manufacturers include an increased demand for ketamine and manufacturing delays. There are currently only two registered ketamine products on the SA market (both of which are generics), which further contributes to the lack of a viable supply chain. In general, generic injectable medications are most commonly affected by shortages, owing to the costly and complex production processes related to their manufacture and the fact that they have smaller profit margins than branded medications, so there is a lack of interest on the part of manufacturers in producing the line. Furthermore, backlogs at the South African Health Products Regulatory Authority with regard to both new medicine and generic medicine registration limit the available alternatives.
In addition to manufacturing issues related to ketamine shortages, logistics and supply chain challenges are contributing factors. Maintaining an adequate inventory in the midst of a drug shortage is a difficult task, leading some providers and organisations to stock up on medicines in anticipation of a shortage, which increases costs and risks wasted inventory. ${ }^{[12]}$ While the National Department of Health is aware of the problem, and there have been several studies attesting to medicine shortages, it is evident that procurement and distribution of medical supplies remain inadequate in SA. ${ }^{[15]}$ Supply issues have also been linked to inadequate training and the inability of personnel to manage medicine supplies, report shortages, and respond appropriately to prevent their recurrence. ${ }^{[16]}$ Additionally, challenges in the healthcare system related to shortages of healthcare workers, weak oversight and management, and inadequate monitoring and evaluation of clinical data have been reported as further contributing factors.

\section{Conclusions}

In a nutshell, ketamine is exceptionally user-friendly for relatively untrained clinicians in resource-limited settings, and any shortage of this drug should therefore be grounds for grave concern. Although there are a small number of alternatives to ketamine for the management of burn pain, the viability of these options in the public health sector is dubious. Unfortunately, the ketamine issue is not an isolated one. Several essential medicines used in surgical patient care and critical care settings in SA are frequently out of stock or in short supply. This article aims to start the conversation and the thinking about identifying reasons for the shortages and finding solutions to this problem to ensure that we have a plan B. The solution will require a multidisciplinary effort from each level of the healthcare system (districts, province and the National Department of Health). Failure to address the ketamine shortage before stocks are completely depleted will have far-reaching effects across our healthcare system.

\section{Declaration. None.}

\section{Acknowledgements. None.}

Author contributions. SW and VB both contributed to the article. Funding. Research reported in this publication was supported by the Fogarty International Center, National Institutes of Health (NIH) Common Fund, Office of Strategic Coordination, Office of the Director (CF/OSC/OD/NIH), Office of AIDS Research, Office of the Director (OAR/OD/NIH), National Institute of Mental Health (NIMH/NIH), award no. D43TW010131. The content is solely the responsibility of the authors and does not necessarily represent the official views of the NIH.

Conflicts of interest. None.

1. Rode H, Berg AM, Rogers A. Burn care in South Africa. Ann Burns Fire Disasters 2011;24(1):7-8. 2. South African Medical Research Council. South African Medical Research Council (SAMRC) presents on the burden of disease: Focus: Violence, peace and injuries. 8 November 2017. http://pmg-assets. s3-website-eu-west-1.amazonaws.com/171108VIOLENCE_PEACE_AND_INJURIES.pdf (accessed 16 March 2019).

3. World Health Organization. Burns. https:/wwwwwho.int/news-room/fact-sheets/detai/burns (accessed 16 March 2019).
(

4. Patterson DR, Hofland HW, Espey K, Sharar S; Nursing Committee of the International Society Patterson DR, Hofland HW, Espey K, Sharar S; Nursing Committee of the International Society
for Burn Injuries. Pain management. Burns 2004;30(8):A10-A15. https://doi.org/10.1016/j. for Burn Injuries. Pain management. Burns 2004;30(8):A10-A15. https://doi.org/10.1016/j.
burns.2004.08.004

Christian LM, Graham JE, Padgett DA, Glaser R, Kiecolt-Glaser JK. Stress and wound healing. Neuroimmunomodulation 2006;13(5-6):337-346. https://doi.org/10.1159/000104862

6. Raymond I, Ancoli-Israel S, Choinière M. Sleep disturbances, pain and analgesia in adults hospitalized for burn injuries. Sleep Med 2004;5(6):551-559. https://doi.org/10.1016/j.sleep.2004.07.007

7. Gao M, Rejaei D, Liu H. Ketamine use in current clinical practice. Acta Pharmacol Sin 2016;37(7):865872. https://doi.org/10.1038/aps.2016.5

8. Salvadore G, Singh JB. Ketamine as a fast acting antidepressant: Current knowledge and open questions. CNS Neurosci Ther 2013;19:428-436. https://doi.org/10.1111/cns.12103

9. Reardon S. Rave drug tested against depression: Companies and clinicians turn to ketamine to treat mental-health disorder as pipeline of new druss dries up. Nature 2015:517:130-131. https:/doi. org/10.1038/517130a 
10. National Department of Health, South Africa. Standard Treatment Guidelines and Essential Medicines List for South Africa: Hospital Level, Adults. 2015. http://www.kznhealth.gov.za/pharmacy/ hospitallevel_adult2015.pdf (accessed 31 October 2019).

11. National Department of Health, South Africa. Standard Treatment Guidelines and Essential Medicines List: Tertiary and Quaternary Level 2013. 2014. http://www.health.gov.za/index.php/standardtreatment-guidelines-and-essential-medicines-list (accessed 31 October 2019).

12. Gales A, Maxwell S. Ketamine: Recent evidence and current uses. Anaesthesia Tutorial of the Week. 12 June 2018. https://www.wfsahq.org/components/com_virtual_library/media/3a6c6301ec0cclfaf50 7f2959aeleala-atow-381-00-01.pdf (accessed 23 October 2019).

13. Treatment Action Campaign, Doctors without Borders/Médecins Sans Frontières, Rural Health Advocacy Project, Rural Doctors Association of Southern Africa, SECTION27, Southern Africa HIV Clinicians Society. Stock Outs in South Africa: Second Annual Report. 2014. https://www.msf.org.za/system/tdf/ drug_stockouts_2014_report_0.pdf?file=1\&type=node\&id=3065\&force $=($ accessed 26 June 2019).
14. Babl FE, Jamison SR, Spicer M, Bernard S. Inhaled methoxyflurane as a prehospital analgesic in children. Emerg Med Australas 2006;18(4):404-410. https://doi.org/10.1111/j.1742-6723. in children.

15. Barrera K, McNicoll CF, Sangii NF. Drug shortages: The invisible epidemic. http://bulletin.facs.

15.
org/2018/11/drug-shortages-the-invisible-epidemic/ (accessed 27 April 2019).
16. Hodes R, Price I, Bungane N, Toska E, Cluver L. How front-line healthcare workers respond . Hodes R, Price I, Bungane N, Toska E, Cluver L. How front-line healthcare workers respond
to stock-outs of essential medicines in the Eastern Cape Province of South Africa. S Afr Med J to stock-outs of essential medicines in the Eastern Cape Province of
2017;107(9):738-740. https://doi.org/10.7196/SAMJ.2017.v107i9.12476

Accepted 29 July 2019. 